\title{
EIGENVALUE DISTRIBUTION OF BIPARTITE LARGE WEIGHTED RANDOM GRAPHS. RESOLVENT APPROACH
}

\author{
V. Vengerovsky, Institute for Low Temperature Physics, Ukraine
}

\begin{abstract}
We study eigenvalue distribution of the adjacency matrix $A^{(N, p, \alpha)}$ of weighted random bipartite graphs $\Gamma=\Gamma_{N, p}$. We assume that the graphs have $N$ vertices, the ratio of parts is $\frac{\alpha}{1-\alpha}$ and the average number of edges attached to one vertex is $\alpha \cdot p$ or $(1-\alpha) \cdot p$. To each edge of the graph $e_{i j}$ we assign a weight given by a random variable $a_{i j}$ with the finite second moment.

We consider the resolvents $G^{(N, p, \alpha)}(z)$ of $A^{(N, p, \alpha)}$ and study the functions $f_{1, N}(u, z)=$ $\frac{1}{[\alpha N]} \sum_{k=1}^{[\alpha N]} e^{-u a_{k}^{2} G_{k k}^{(N, p, \alpha)}(z)}$ and $f_{2, N}(u, z)=\frac{1}{N-[\alpha N]} \sum_{k=[\alpha N]+1}^{N} e^{-u a_{k}^{2} G_{k k}^{(N, p, \alpha)}(z)}$ in the limit $N \rightarrow$ $\infty$. We derive closed system of equations that uniquely determine the limiting functions $f_{1}(u, z)$ and $f_{2}(u, z)$. This system of equations allow us to prove the existence of the limiting measure $\sigma_{p, \alpha}$. The weak convergence in probability of normalized eigenvalue counting measures is proved.
\end{abstract}

\section{Introduction}

The spectral theory of graphs is an actively developing field of mathematics involving a variety of methods and deep results (see the monographs [5, 6, 11]). Given a graph with $N$ vertices, one can associate with it many different matrices, but the most studied are the adjacency matrix and the Laplacian matrix of the graph. Commonly, the set of $N$ eigenvalues of the adjacency matrix is referred to as the spectrum of the graph. In these studies, the dimension of the matrix $N$ is usually regarded as a fixed parameter. The spectra of infinite graphs is considered in certain particular cases of graphs having one or another regular structure (see for example [13]).

Another large class of graphs, where the limiting transition $N \rightarrow \infty$ provides a natural approximation is represented by random graphs [3, 12. In this branch, geometrical and topological properties of graphs (e.g. number of connected components, size of maximal connected component) are studied for immense number of random graph ensembles. One of the classes of the prime reference is the binomial random graph originating by P. Erdôs (see, e.g. [12]). Given a number $p_{N} \in(0,1)$, this family of graphs $\mathbf{G}\left(N, p_{N}\right)$ is defined by taking as $\Omega$ the set of all graphs on $N$ vertices with the probability

$$
P(G)=p_{N}^{e(G)}\left(1-p_{N}\right)^{\left(\begin{array}{c}
N \\
2
\end{array}\right)-e(G)},
$$

where $e(G)$ is the number of edges of $G$. Most of the random graphs studies are devoted to the cases where $p_{N} \rightarrow 0$ as $N \rightarrow \infty$.

Intersection of these two branches of the theory of graphs contains the spectral theory of random graphs that is still poorly explored. However, a number of powerful tools can be employed here, because the ensemble of random symmetric $N \times N$ adjacency matrices $A_{N}$ is a particular representative of the random matrix theory, where the limiting transition $N \rightarrow \infty$ is intensively studied during half of century since the pioneering works by E. Wigner [21]. Initiated 
by theoretical physics applications, the spectral theory of random matrices has revealed deep nontrivial links with many fields of mathematics.

Spectral properties of random matrices corresponding to (1.1) were examined in the limit $N \rightarrow \infty$ both in numerical and theoretical physics studies [7, 8, 9, 17, 18, 19]. There are two major asymptotic regimes: $p_{N} \gg 1 / N$ and $p_{N}=O(1 / N)$ and corresponding models can be called the diluted random matrices and sparse random matrices, respectively. The first studies of spectral properties of sparse and diluted random matrices in the physical literature are related with the works [18, [19, [17, where equations for the limiting density of states of sparse random matrices were derived. In papers [17] and [10] a number of important results on the universality of the correlation functions and the Anderson localization transition were obtained. But all these results were obtained with non rigorous replica method.

On mathematical level of rigour, the eigenvalue distribution of dilute random matrices was studied in [15. It was shown that the normalized eigenvalue counting function of

$$
\frac{1}{\sqrt{N p_{N}}} A_{N, p_{N}}
$$

converges in the limit $N, p_{N} \rightarrow \infty$ to the distribution known as the semicircle or Wigner law [21]. The moments of this distribution verify well-known recurrent relation for the Catalan numbers and can be found explicitly. Therefore one can say that the dilute random matrices represent explicitly solvable model (see also [18, 19]).

In the series of papers [2, 4, 3] and in [14, the adjacency matrix and the Laplace matrix of random graphs (1.1) with $p_{N}=p N$ were studied. It was shown that this sparse random matrix ensemble can also be viewed as the explicitly solvable model.

In the present paper we consider a bipartite analogue of large sparse random graph. This article follows the method of [14. The moment method for this problem was considered in [20].

\section{Main results}

We can introduce the randomly weighted adjacency matrix of random bipartite graphs. Let $\Xi=\left\{a_{i j}, i \leq j, i, j \in \mathbb{N}\right\}$ be the set of jointly independent identically distributed (i.i.d.) random variables determined on the same probability space. We set $a_{j i}=a_{i j}$ for $i \leq j$.

Given $0<p \leq N$, let us define the family $D_{N}^{(p)}=\left\{d_{i j}^{(N, p)}, i \leq j, i, j \in \overline{1, N}\right\}$ of jointly independent random variables

$$
d_{i j}^{(N, p)}= \begin{cases}1, & \text { with probability } p / N \\ 0, & \text { with probability } 1-p / N\end{cases}
$$

We set $d_{j i}=d_{i j}$ and assume that $D_{N}^{(p)}$ is independent from $\Xi$.

Let $\alpha \in(0,1)$, define $I_{\alpha, N}=\overline{1,[\alpha \cdot N]}$, where $[\cdot]$ is an integer part of the number. Now one can consider the real symmetric $N \times N$ matrix $A^{(N, p, \alpha)}(\omega)$ :

$$
\left[A^{(N, p, \alpha)}\right]_{i j}= \begin{cases}a_{i j} \cdot d_{i j}^{(N, p)}, & \text { if }\left(i \in I_{\alpha, N} \wedge j \notin I_{\alpha, N}\right) \vee\left(i \notin I_{\alpha, N} \wedge j \in I_{\alpha, N}\right), \\ 0, & \text { otherwise }\end{cases}
$$

that has $N$ real eigenvalues $\lambda_{1}^{(N, p, \alpha)} \leq \lambda_{2}^{(N, p, \alpha)} \leq \ldots \leq \lambda_{N}^{(N, p, \alpha)}$.

The normalized eigenvalue counting function of $A^{(N, p, \alpha)}$ is determined by the formula

$$
\sigma\left(\lambda ; A^{(N, p, \alpha)}\right)=\frac{\sharp\left\{j: \lambda_{j}^{(N, p, \alpha)}<\lambda\right\}}{N} .
$$


We denote by $\mathcal{F}$ the class of functions which are analytic with respect to $z: \Re z>0$ and for any fixed $z: \Re z>0$ possessing the norm

$$
\|f(u, z)\|=\sup _{u>0} \frac{|f(u, z)|}{\sqrt{1+u}} .
$$

Theorem 1. Assume that $\mu(a)=\mathbb{E}\left\{\theta\left(a-a_{i, j}\right)\right\}$ the probability distribution of $a_{i, j}$ possesses the property

$$
\int a^{4} d \mu(a)=X_{4}<\infty
$$

(We denote by $X_{i}$ i absolute moment of a, i.e. $X_{i}=\int|a|^{i} d \mu(a)$.) Then the measure $\sigma\left(\lambda ; A^{(N, p, \alpha)}\right)$ converges weakly in probability to nonrandom measure $\sigma_{p, \alpha}$

$$
\sigma\left(\cdot ; A^{(N, p, \alpha)}\right) \stackrel{w, P}{\rightarrow} \sigma_{p, \alpha}, N \rightarrow \infty .
$$

The Stieltjes transform $g_{\sigma_{p, \alpha}}$ of the limiting measure $\sigma_{p, \alpha}$ can be found as follows:

$$
\begin{gathered}
g_{\sigma_{p, \alpha}}(z)=-i h(i z) \\
h(z): \mathbb{C}_{+} \rightarrow \mathbb{C}_{+}, h(z)=\alpha \cdot h_{1}(z)+(1-\alpha) \cdot h_{2}(z) \\
h_{i}(z)=-\left.X_{2}^{-1} \frac{\partial}{\partial u} f_{i}(u, z)\right|_{u=0}, i=1,2,
\end{gathered}
$$

where a pair $f_{1}(u, z)$ and $f_{2}(u, z)$ is a unique solution of the following system of functional equations in the class $\mathcal{F}$ :

$$
\left\{\begin{array}{l}
f_{1}(u, z)=L\left(f_{2}, \mu, 1-\alpha\right) \\
f_{2}(u, z)=L\left(f_{1}, \mu, \alpha\right)
\end{array}\right.
$$

where

$$
L(f, \mu, \alpha)=1-u^{1 / 2} e^{-\alpha p} \int|a| d \mu(a) \int_{0}^{\infty} d v \frac{\mathcal{J}_{1}(2|a| \sqrt{u v})}{\sqrt{v}} \exp \{-z v+\alpha p f(v, z)\},
$$

$\mathcal{J}_{1}(\zeta)$ is the Bessel function:

$$
\mathcal{J}_{1}(\zeta)=\frac{\zeta}{2} \sum_{k=0}^{\infty} \frac{\left(-\zeta^{2} / 4\right)^{k}}{k !(k+1) !}
$$

Proposition 1. Condition 2.4) in Theorem 1 could be replaced by

$$
\int a^{2} d \mu(a)=X_{2}<\infty
$$

via truncation procedure.

The proof of Proposition 1 is given in section 4 .

Theorem 1 is a corollary of Theorem 2 .

Theorem 2. Let the distribution of $a_{j, k}$ satisfy condition (2.4). Then (i) the variance of $g_{N, p, \alpha}(z)$ vanishes in the limit $N \rightarrow \infty$ :

$$
\mathbb{E}\left\{\left|g_{N, p, \alpha}(z)-\mathbb{E}\left\{g_{N, p, \alpha}(z)\right\}\right|^{2}\right\} \leq \frac{C\left(z, p, \alpha, X_{2}\right)}{N^{1 / 2}},
$$

(ii) there exists the limiting probability measure $\sigma_{p, \alpha}$ such that

$$
g_{\sigma_{p, \alpha}}(z)=\lim _{N \rightarrow \infty} \mathbb{E}\left\{g_{N, p, \alpha}(z)\right\}=-i h(i z),
$$

for arbitrary compact in $\mathbb{C}_{+}$the convergence is uniform, and the function $h(z): \mathbb{C}_{+} \rightarrow \mathbb{C}_{+}$can be expressed in terms of the pair of functions $f_{1}(u, z)$ and $f_{2}(u, z)$ (see (2.7)-(2.8)), which is a unique solution of the system of functional equations (2.9) in the class $\mathcal{F}$. 


\section{Proof of Theorem 1}

For any $z: \Re z>0$ consider the functions $f_{1, N}(u, z): \mathbb{R}_{+} \rightarrow \mathbb{C}, f_{2, N}(u, z): \mathbb{R}_{+} \rightarrow \mathbb{C}$ :

$$
\begin{gathered}
f_{1, N}(u, z)=\frac{1}{[\alpha N]} \sum_{k=1}^{[\alpha N]} e^{-u a_{k}^{2} G_{k k}^{(N, p, \alpha)}(z)}, \quad G^{(N, p, \alpha)}(z)=\left(z-i A^{(N, p, \alpha)}\right)^{-1} \\
f_{2, N}(u, z)=\frac{1}{N-[\alpha N]} \sum_{k=[\alpha N]+1}^{N} e^{-u a_{k}^{2} G_{k k}^{(N, p, \alpha)}(z)}
\end{gathered}
$$

where $\left\{a_{i}\right\}_{i=1}^{\infty}$ is a family of i.i.d. random variables which do not depend on $\left\{a_{i, j}\right\}_{i<j}^{\infty}$ and have

the same probability distribution as $a_{1,2}$. It is easy to see that $G_{N N}^{(N, p, \alpha)}(z)$ can be represented in the form

$$
G_{N N}^{(N, p)}(z)=\left(z-i A_{N N}^{(N, p, \alpha)}+\sum_{j, k=1}^{N-1} \widetilde{G}_{j k}^{(N-1, p, \alpha)} A_{N j}^{(N, p, \alpha)} A_{N k}^{(N, p, \alpha)}\right)^{-1},
$$

where the matrix $\left\{\widetilde{G}_{i j}^{(N-1, p, \alpha)}(z)\right\}_{i, j=2}^{N}$ is a resolvent of the matrix $i \widetilde{A}^{(N-1, p, \alpha)}$, which can be obtained from $A^{(N, p, \alpha)}$ by deleting the last column and the last row. Let us use the formula (see [1]):

$$
e^{-u a^{2} R}=1-u^{1 / 2}|a| \int_{0}^{\infty} d v \frac{\mathcal{J}_{1}(2|a| \sqrt{u v})}{\sqrt{v}} \exp \left\{-R^{-1} v\right\}
$$

which is valid for any $u \geq 0, \Re R>0$. Then, on the basis of (3.2), we get

$$
\begin{aligned}
\exp \left\{-u a_{N}^{2} G_{N N}^{(N, p, \alpha)}\right\}= & 1-u^{1 / 2}\left|a_{N}\right| \int_{0}^{\infty} d v \frac{\mathcal{J}_{1}\left(2\left|a_{N}\right| \sqrt{u v}\right)}{\sqrt{v}} \\
& \exp \left\{-z v-v \sum_{j, k=1}^{[\alpha N]} \widetilde{G}_{i j}^{(N-1, p, \alpha)} A_{N i}^{(N, p, \alpha)} A_{N j}^{(N, p, \alpha)}\right\} .
\end{aligned}
$$

Denote

$$
R_{N}(z)=\sum_{j, k=1, j \neq k}^{[\alpha N]} \widetilde{G}_{j k}^{(N-1, p)} A_{N j}^{(N, p, \alpha)} A_{N k}^{(N, p, \alpha)}
$$

Proposition 2.

$$
\mathbb{E}\left\{\left|R_{1}(z)\right|^{2}\right\} \leq 2 \frac{p^{2} X_{2}^{2}}{N|\Re z|^{2}}+\frac{p^{4} X_{1}^{4}}{N^{2}|\Re z|^{2}}+4 \frac{p^{3} X_{1}^{2} X_{2}}{N^{2}|\Re z|^{2}}+6 \frac{p^{4} X_{1}^{4}}{N|\Re z|^{2}}+8 \frac{p^{3} X_{1}^{2} X_{2}}{N|\Re z|^{2}},
$$

where

$$
X_{k}=\int|a|^{k} d \mu(a)
$$

Proof.

$$
\begin{aligned}
& \mathbb{E}\left\{\left|R_{1}(z)\right|^{2}\right\}=\sum_{\neq\left\{j_{1}, j_{2}, k_{1}, k_{2}\right\}}^{[\alpha N]} \mathbb{E}\left\{\widetilde{G}_{j_{1} k_{1}}^{(N-1, p)} \overline{\widetilde{G}_{j_{2} k_{1}}^{(N-1, p)}} A_{N j_{1}}^{(N, p)} A_{N j_{2}}^{(N, p)} A_{N k_{1}}^{(N, p)} A_{N k_{2}}^{(N, p)}\right\} \\
& +4 \sum_{\neq\left\{j, k_{1}, k_{2}\right\}}^{[\alpha N]} \mathbb{E}\left\{\widetilde{G}_{j k_{1}}^{(N-1, p)} \overline{\widetilde{G}_{j k_{1}}^{(N-1, p)}}\left|A_{N j}^{(N, p)}\right|^{2} A_{N k_{1}}^{(N, p)} A_{N k_{2}}^{(N, p)}\right\} \\
& +2 \sum_{j \neq k}^{[\alpha N]} \mathbb{E}\left\{\widetilde{G}_{j k}^{(N-1, p)} \overline{\widetilde{G}_{j k}^{(N-1, p)}}\left|A_{N j}^{(N, p)}\right|^{2}\left|A_{N k}^{(N, p)}\right|^{2}\right\}=H_{1}+4 H_{2}+2 H_{3} .
\end{aligned}
$$


Averaging with respect to $\left\{A_{N, i}^{(N, p)}\right\}_{i=1}^{N-1}$ and using the fact that $\left\{\widetilde{G}_{i j}^{(N-1, p)}(z)\right\}_{i, j=1}^{N-1}$ do not depend on $A_{N, i}^{(N, p)}$, we obtain

$$
\begin{aligned}
& H_{1} \leq X_{1}^{4} \frac{p^{2}}{N^{2}} \mathbb{E}\left\{\left|N^{-1} \sum_{j, k}^{[\alpha N]} \widehat{G}_{j k}\right|^{2}\right\}+6 \frac{p^{4} X_{1}^{4}}{N|\Re z|^{2}} \leq \frac{p^{2} X_{1}^{4}}{N^{2}|\Re z|^{2}}+6 \frac{p^{4} X_{1}^{4}}{N|\Re z|^{2}} \\
& H_{2} \leq X_{1}^{2} X_{2} \frac{p}{N^{3}} \sum_{k_{1} \neq k_{2}}^{[\alpha N]} \mathbb{E}\left\{\left[\widehat{G} \widehat{G}^{*}\right]_{k_{1} k_{2}}\right\}+2 \frac{p^{3} X_{1}^{2} X_{2}}{N|\Re z|^{2}} \leq \frac{p X_{1}^{2} X_{2}}{N^{2}|\Re z|^{2}}+2 \frac{p^{3} X_{1}^{2} X_{2}}{N|\Re z|^{2}}, \\
& H_{3} \leq \frac{X_{2}^{2}}{N^{2}} \sum_{k}^{[\alpha N]} \mathbb{E}\left\{\left[\widehat{G} \widehat{G}^{*}\right]_{k k}\right\} \leq \frac{X_{2}^{2}}{N|\Re z|^{2}} .
\end{aligned}
$$

Besides, since evidently

$$
\Re\left\{\sum \widetilde{G}_{i j}^{(N-1, p)} A_{N i}^{(N, p, \alpha)} A_{N j}^{(N, p, \alpha)}\right\} \geq 0, \quad \Re\left\{\sum \widetilde{G}_{j j}^{(N-1, p, \alpha)}\left|A_{j j}^{(N, p, \alpha)}\right|^{2}\right\} \geq 0,
$$

the inequality

$$
\left|e^{-z_{1}}-e^{-z_{2}}\right| \leq\left|z_{1}-z_{2}\right| \quad\left(\Re z_{1}, \Re z_{2} \geq 0\right)
$$

and (3.4) imply

$$
\begin{aligned}
\mathbb{E} \exp \left\{-u a_{N}^{2} G_{N N}^{(N, p, \alpha)}\right\}= & 1-u^{1 / 2} \mathbb{E}\left|a_{N}\right| \int_{0}^{\infty} d v \frac{\mathcal{J}_{1}\left(2\left|a_{N}\right| \sqrt{u v}\right)}{\sqrt{v}} \\
& \mathbb{E}_{2} \exp \left\{-z v-v \sum_{i=1}^{[\alpha N]} \widetilde{G}_{i i}^{(N-1, p, \alpha)}\left|A_{N j}^{(N, p, \alpha)}\right|^{2}\right\}+\widetilde{r}_{N}(u),
\end{aligned}
$$

where $\mathbb{E}_{2}$ denotes averaging over $\left\{a_{i j}\right\}_{i, j}$ and $\left\{d_{i j}^{(N, p)}\right\}_{i, j}$. Remainder $\widetilde{r}_{N}(u)$ obeys the following condition

$$
\left|\widetilde{r}_{N}(u)\right| \leq \mathbb{E}\left|R_{1}\right| u^{1 / 2} \mathbb{E}\left|a_{N}\right| \int_{0}^{\infty} d v\left|\frac{\mathcal{J}_{1}\left(2\left|a_{1}\right| \sqrt{u v}\right)}{\sqrt{v}} e^{-z v}\right| \leq C\left|R_{1}\right| u^{1 / 2} \mathbb{E}\left|a_{N}\right||\Re z|^{-1 / 2} .
$$

In the last inequality we use the estimate for the Bessel function

$$
\left|\mathcal{J}_{1}(u)\right| \leq 1 .
$$

Here and below we denote by $C$ some constants (different in different formulas), which do not depend on $N, z, p, \alpha$. Taking into account (3.6), we get

$$
\left|\widetilde{r}_{N}(u)\right| \leq \frac{C\left(p, X_{2}\right) u^{1 / 2}}{\sqrt{N}|\Re z|^{5 / 2}} .
$$

Since $\widetilde{G}^{(N-1, p)}(z)$ does not depend on $\left\{d_{N, j}^{(N, p)}\right\}_{j=1}^{N}$, we obtain

$$
\begin{aligned}
\mathbb{E}_{2} \exp \left\{-v \sum_{i=1}^{[\alpha N]} \widetilde{G}_{i i}^{(N-1, p, \alpha)}\left|A_{N j}^{(N, p, \alpha)}\right|^{2}\right\} & =\mathbb{E}\left\{\prod_{j=1}^{[\alpha N]}\left(\left(1-\frac{p}{N}\right)+\frac{p}{N} e^{-v a_{N j}^{2} \widetilde{G}_{j j}^{(N-1, p)}}\right)\right\} \\
& =e^{-\alpha p} \mathbb{E}\left\{\exp \left\{\alpha p \widetilde{f}_{1, N-1}(v, z)\right\}\right\}+R_{N}(v)
\end{aligned}
$$


where

$$
\widetilde{f}_{1, N-1}(u, z)=\frac{1}{[\alpha N]} \sum_{k=1}^{[\alpha N]} e^{-v a_{N j}^{2} \widetilde{G}_{k k}^{(N, p, \alpha)}(z)},
$$

and $R_{N}$ satisfy the estimate

$$
\left|R_{N}(v)\right| \leq \frac{C p^{2}}{N}
$$

Using (3.8), we get

$$
\begin{aligned}
\left|\mathbb{E} \exp \left\{\alpha p \tilde{f}_{1, N-1}(v, z)\right\}-\exp \left\{\alpha p \mathbb{E} \tilde{f}_{1, N-1}(v, z)\right\}\right| & \\
& \leq \alpha p e^{\alpha p} \mathbb{E}\left|\widetilde{f}_{1, N-1}(v, z)-\mathbb{E} \widetilde{f}_{1, N-1}(v, z)\right|
\end{aligned}
$$

Further considerations are based on the Lemma 1

Lemma 1. Fix $\alpha \in(0,1)$. Let $A^{(n)}$ be a real symmetric $n \times n$ matrix, such that

$$
A_{i j}^{(n)}= \begin{cases}a_{i j}, & \text { if }\left(i \in I_{\alpha, n} \wedge j \notin I_{\alpha, n}\right) \vee\left(i \notin I_{\alpha, n} \wedge j \in I_{\alpha, n}\right), \\ 0, & \text { otherwise }\end{cases}
$$

where $\left\{a_{i j}\right\}_{1 \leq i \leq j}$ is a family of jointly independent identically distributed random variables that obey the following conditions

$$
\mathbb{E}\left|a_{i j}\right| \leq \frac{C}{n}, \mathbb{E} a_{i j}^{2} \leq \frac{C}{n}
$$

For $z: \Re z>0$ consider

$$
R=(z-i A)^{-1}, \quad F_{n}(z)=[\alpha \cdot n]^{-1} \sum_{j=1}^{[\alpha \cdot n]} \varphi_{j}\left(R_{j j}\right),
$$

where random functions $\varphi_{j}$ satisfy the following condition

$$
\left|\varphi_{j}^{\prime}(\zeta)\right| \leq C_{3} \cdot \alpha_{j}
$$

where $\left\{\alpha_{j}\right\}$ is a set of jointly independent identically distributed random variables also independent of $\left\{a_{i j}\right\}$ such that $\mathbb{E}\left\{\alpha_{1}^{2}\right\}<\infty$. Then

$$
\operatorname{Var}_{n}(z) \leq \frac{4(1-\alpha) C_{3}^{2}}{\alpha n|\Re z|^{4}} \cdot \mathbb{E}\left\{\alpha_{1}^{2}\right\} \cdot\left(n \mathbb{E}\left|a_{12}\right|+\left(n \mathbb{E} a_{12}^{2}\right)^{2}\right) .
$$

The proof of Lemma 1 is given in section 4

Remark 1. Lemma 1 is still valid for $F_{n}(z)=n^{-1} \sum_{j=1}^{n} \varphi_{j}\left(R_{j j}\right)$ with changed constants.

Lemma 1 for $\varphi(\zeta)=\exp \left\{-v a_{N j}^{2} \zeta\right\}, \alpha_{j}=v a_{N j}^{2}, C_{3}=1, n=N-1$ implies

$$
\mathbb{E}\left|\widetilde{f}_{1, N-1}(v, z)-\mathbb{E} \widetilde{f}_{1, N-1}(v, z)\right|^{2} \leq \frac{\widetilde{C}^{2}\left(X_{4}, p\right) v^{2}}{\alpha N|\Re z|^{4}}
$$

Relations (3.13) and (3.19) yield

$$
\left|\mathbb{E} \exp \left\{\alpha p \widetilde{f}_{1, N-1}(v, z)\right\}-\exp \left\{\alpha p \mathbb{E} \widetilde{f}_{1, N-1}(v, z)\right\}\right| \leq \alpha p e^{\alpha p} \frac{\widetilde{C}\left(X_{4}, p\right) v}{\alpha^{1 / 2} N^{1 / 2}|\Re z|^{2}}
$$

Combining (3.4)-(3.20), we get

$$
\mathbb{E} f_{2, N}(u, z)=1-u^{1 / 2} e^{-\alpha p} \int_{0}^{\infty} d v e^{-z v} \frac{\mathcal{J}_{1}(2 \sqrt{u v})}{\sqrt{v}} e^{\alpha p \mathbb{E} \widetilde{f}_{1, N-1}(v, z)}+r(u),
$$




$$
r(u) \leq \frac{\widetilde{C}\left(\mathbb{E} a_{N j}^{4}, p\right) u^{1 / 2}}{N^{1 / 2}|\Re z|^{7 / 2}} .
$$

In order to get the closed system of equations, we have to replace $\widetilde{f}_{1, N-1}$ by $f_{1, N}$. To this purpose we use the next bound on their difference

$$
\left|\mathbb{E} f_{1, N}(v, z)-\mathbb{E} \tilde{f}_{1, N-1}(v, z)\right| \leq \frac{\widetilde{C}\left(X_{4}, p\right) v}{\alpha N^{1 / 2}|\Re z|^{2}} .
$$

Indeed, using Lemma 1 for $\varphi(\zeta)=\exp \left\{-v a_{j}^{2} \zeta\right\}, \alpha_{j}=v a_{j}^{2}, C_{3}=1, n=N$, we get

$$
\mathbb{E}\left|f_{1, N}(v, z)-\mathbb{E} f_{1, N}(v, z)\right|^{2} \leq \frac{\widetilde{C}^{2}\left(X_{4}, p\right) v^{2}}{\alpha N|\Re z|^{4}} .
$$

Combining (3.23), (3.19) and (4.6) for $\varphi(\zeta)=\exp \left\{-v a_{N j}^{2} \zeta\right\}, \alpha_{j}=v a_{N j}^{2}, C_{3}=1, n=N$, we obtain (3.22).

The inequalities (3.22), (3.8) and (3.21) imply

$$
\begin{gathered}
\mathbb{E} f_{2, N}(u, z)=1-u^{1 / 2} e^{-\alpha p} \int_{0}^{\infty} d v e^{-z v} \frac{\mathcal{J}_{1}(2 \sqrt{u v})}{\sqrt{v}} e^{\alpha p \mathbb{E} f_{1, N}(v, z)}+r(u), \\
r(u) \leq \frac{\widetilde{C}\left(X_{4}, p\right) u^{1 / 2}}{N^{1 / 2}|\Re z|^{7 / 2}} .
\end{gathered}
$$

Let us consider the Banach space $\mathcal{H}$ of all the functions $h: \mathbb{R}_{+} \rightarrow \mathbb{C}$ which possess the norm (2.3). $\mathcal{H}^{2}$ possess the norm $\left\|\left(h_{1}, h_{2}\right)\right\|_{\mathcal{H}^{2}}=\max \left\{\left\|h_{1}\right\|_{\mathcal{H}},\left\|h_{2}\right\|_{\mathcal{H}}\right\}$.

Define the operator $F_{z}: \mathcal{H}^{2} \rightarrow \mathcal{H}^{2}$ of the form

$$
F_{z}\left(\varphi_{1}, \varphi_{2}\right)=\left(\psi_{1}, \psi_{2}\right), \psi_{1}(u)=L\left(f_{2}, \mu, 1-\alpha\right), \psi_{2}(u)=L\left(f_{1}, \mu, \alpha\right) .
$$

Let us denote by $B_{0,2}=\left\{h \in \mathcal{H}^{2},\|h\|_{\mathcal{H}^{2}} \leq 2\right\}$ the ball of radius 2 centered in the origin. Then for any $\varphi_{1}, \varphi_{2}:\left\|\varphi_{1}\right\| \leq 2,\left\|\varphi_{2}\right\| \leq 2$

$$
\left\|F_{z}\left(\varphi_{1}\right)-F_{z}\left(\varphi_{2}\right)\right\| \leq X_{1} p e^{2 p+\frac{2 p^{2}}{|\Re z|}}\left(\frac{2}{|\Re z|}+\frac{4}{|\Re z|^{1 / 2}}\right)\left\|\varphi_{1}-\varphi_{2}\right\| .
$$

Indeed, inequalities (3.8), (3.10) imply

$$
\left\|F_{z}\left(\varphi_{1}\right)-F_{z}\left(\varphi_{2}\right)\right\|_{\mathcal{H}^{2}} \leq X_{1} p\left\|\varphi_{1}-\varphi_{2}\right\| \int_{0}^{\infty} \frac{d v\left(1+v^{1 / 2}\right) e^{-|\Re z| v+2 p\left(1+v^{1 / 2}\right)}}{\sqrt{v}}
$$

Using the trivial inequality

$$
2 p v^{1 / 2}-v|\Re z| / 2 \leq 2 \frac{p^{2}}{|\Re z|},
$$

we get (3.26).

It is easy to see that $\left\|F_{z}(0)\right\|$ obey the inequality

$$
\left\|F_{z}(0)\right\|_{\mathcal{H}^{2}} \leq \sup _{u>0} \frac{1+2 X_{1}|\Re z|^{-1 / 2} u^{1 / 2}}{1+u^{1 / 2}} \leq 1+2 X_{1}|\Re z|^{-1 / 2} .
$$

So there is $M>0$ such that

$$
\left\|F_{z}\left(\varphi_{1}\right)-F_{z}\left(\varphi_{2}\right)\right\|<1 / 4\left\|\varphi_{1}-\varphi_{2}\right\|, \quad\left\|F_{z}(0)\right\| \leq \frac{5}{4}, \quad z \in L(M),
$$


where $L(M)=\{z:|\Re z|>M\}$.

Therefore $F_{z}: B_{0,2} \rightarrow B_{0,2}$ and $\left.F_{z}\right|_{B_{0,2}}$ is a contraction mapping for all $z \in L(M)$. Hence, there exists the unique fixed point $f(u, z)$, which is a solution of (2.9). Since $\left|\mathbb{E} f_{1, N}(u, z)\right| \leq$ $1,\left|\mathbb{E} f_{2, N}(u, z)\right| \leq 1, \mathbb{E} f_{N}(u, z) \in B_{0,2}$. (3.30) imply the estimates

$$
\mathbb{E} f_{N}(u, z)=F_{z}\left(\mathbb{E} f_{N}(u, z)\right)+r_{N}(u, z)=\ldots=f(u, z)+r_{N}^{\infty}(u, z),
$$

where

$$
\left\|r_{N}^{\infty}(u, z)\right\| \leq\left\|r_{N}(u, z)\right\| \sum_{k=0}^{\infty} \frac{1}{4^{k}} \leq \frac{4 C(p, M)}{3 N^{1 / 2}}, \quad z \in L(M) .
$$

Hence, $\mathbb{E} f_{N}(u, z) \rightrightarrows f(u, z), \quad z \in L(M)$. Fix u. Since $\mathbb{E} f_{1, N}(u, z), \mathbb{E} f_{2, N}(u, z)$ are analytic and uniformly bounded for arbitrary $\Pi_{\varepsilon, a}=\{z: \varepsilon \leq \Re z \leq 2 M,|\Im z| \leq a\}$, by the Arzela theorem we can choose a subsequence $\left\{N_{k}\right\}_{k=1}^{\infty}$ such that $\mathbb{E} f_{1, N_{k}}(u, z) \rightrightarrows \widetilde{f}_{1}^{(a, \varepsilon)}(u, z), \mathbb{E} f_{2, N_{k}}(u, z) \rightrightarrows$ $\widetilde{f}_{2}^{(a, \varepsilon)}(u, z)$ in $\Pi_{\varepsilon, a}$. Then $f_{1}^{(a, \varepsilon)}(u, z), f_{2}^{(a, \varepsilon)}(u, z)$ are analytic in $\Pi_{\varepsilon, a}$. But

$$
f_{2}^{(a, \varepsilon)}(u, z)=f_{2}(u, z), f_{1}^{(a, \varepsilon)}(u, z)=f_{1}(u, z), \quad|\Re z|>M .
$$

The uniqueness theorem of complex analysis and an arbitrariness of choosing subsequence imply the existence of the analytic extension of $f_{1}(u, z)\left(f_{2}(u, z)\right.$ in $\mathbb{C}_{+}$and uniform convergence in $z f_{\alpha, N}(u, z) \rightrightarrows f_{\alpha}(u, z)$ for arbitrary compact in $\mathbb{C}_{+}$. Thus, if we fixed any $z: \Re z>0$, we obtain that $f_{\alpha, N}(u, z),(\alpha=1,2)$ as a function of $u$ converges pointwise to $f_{\alpha, N}(u, z)$. But since $\left|\frac{\partial}{\partial u} f_{\alpha, N}(u, z)\right| \leq C$ and $\left|f_{\alpha, N}(u, z)\right| \leq 1$, the pointwise convergence imply also the convergence in the norm (2.3). Then, using Lebesgue's dominated convergence theorem, we prove that $f(u, z)=F_{z}(f)(u, z)$ in $\mathbb{C}_{+}$.

Indeed, using Lemma 1 for $\varphi(\zeta)=\zeta, \alpha_{j}=1, C_{3}=1, n=N$, we get (2.12).

Uniform convergence $f_{N}(u, z)$ in $u \in[0,1]$ imply

$$
\begin{aligned}
\mathbb{E} g_{N, p, \alpha}=-i\left(\mathbb{E} a_{1}^{2}\right)^{-1} & \frac{1}{N} \sum_{k=1}^{N} \mathbb{E} a_{k}^{2} \mathbb{E}\left\{G_{k k}^{(N, p, \alpha)}\right\}= \\
& =i\left(\mathbb{E} a_{1}^{2}\right)^{-1}\left(\left.\alpha \frac{\partial}{\partial u} \mathbb{E} f_{1, N}(u, z)\right|_{u=0}+\left.(1-\alpha) \frac{\partial}{\partial u} \mathbb{E} f_{2, N}(u, z)\right|_{u=0}\right),
\end{aligned}
$$

The next simple proposition allows us to make a final step.

Proposition 3. Set $\Psi_{n}(u)=\frac{f_{n}(u)-f_{n}(0)}{u}-f_{n}^{\prime}(0)$. Assume that

$$
\left|\Psi_{n}(u)\right| \leq \varepsilon(u), \varepsilon(u) \rightarrow 0, \text { as } u \rightarrow 0 .
$$

If there exists $f(u)=\lim _{n \rightarrow \infty} f_{n}(u)$ and the function $f$ is differential at $u=0$, then

$$
\lim _{n \rightarrow \infty} f_{n}^{\prime}(0)=f^{\prime}(0)
$$


Proof.

$$
\begin{aligned}
& \mid \frac{f_{1, n}(u, z)-f_{1, n}(0, z)}{u}-\left.\frac{\partial}{\partial u} f_{1, n}(u, z)\right|_{u=0} \mid= \\
&=\left|\int_{0}^{1}\left(\frac{\partial}{\partial u} f_{1, n}(t u, z)-\left.\frac{\partial}{\partial u} f_{1, n}(u, z)\right|_{u=0}\right) d t\right| \leq \\
& \leq \frac{1}{[\alpha N]} \sum_{k=1}^{[\alpha N]} \int_{0}^{1} a_{k}^{2}\left|G_{k k}^{(N, p, \alpha)}\right|\left|e^{-u t a_{k}^{2} G_{k k}^{(N, p, \alpha)}}-1\right| d t d \mu\left(a_{k}\right) \leq \\
& \leq \frac{1}{|\Re z|} \frac{1}{[\alpha N]} \sum_{k=1}^{[\alpha N]}\left(\int_{\left|a^{2}\right|>u^{-1 / 2}}+\int_{\left|a^{2}\right| \leq u^{-1 / 2}}\right) \int a^{2}\left|e^{-u t a_{k}^{2} G_{k k}^{(N, p, \alpha)}}-1\right| d \mu\left(a_{k}\right) d t \leq \\
& \leq \frac{1}{|\Re z|}\left(2 \int_{\left|a^{2}\right|>u^{-1 / 2}} a^{2} d \mu(a)+\frac{1}{|\Re z|} \sqrt{u} \int a^{2} d \mu(a)\right) \stackrel{u \rightarrow 0}{\longrightarrow} 0 .
\end{aligned}
$$

Similar estimate with $f_{2, n}$ is valid too. Hence,

$$
g_{p, \alpha}(z)=\lim _{N \rightarrow \infty} \mathbb{E} g_{N, p, \alpha}(z)=i\left(\mathbb{E} a_{1}^{2}\right)^{-1}\left(\left.\alpha \frac{\partial}{\partial u} f_{1}(u, z)\right|_{u=0}+\left.(1-\alpha) \frac{\partial}{\partial u} f_{2}(u, z)\right|_{u=0}\right)
$$

\section{Proofs of auxiliary statements}

\section{Proof of Lemma 1 .}

Let us denote by $\mathbb{E}_{k}$ averaging over $\left\{a_{i j}\right\}_{i \leq j} i \leq k\left(\mathbb{E}_{n}=\mathbb{E}, \mathbb{E}_{0}\right.$ means absence of averaging), by $n_{1}=[\alpha \cdot n]$, by $n_{2}=n-n_{1}$. Then

$$
\begin{aligned}
& F_{n}-\mathbb{E} F_{n}=\sum_{k=0}^{n_{1}-1}\left(\mathbb{E}_{k} F_{n}-\mathbb{E}_{k+1} F_{n}\right) \Rightarrow \quad \mathbb{E}\left|F_{n}-\mathbb{E} F_{n}\right|^{2}= \\
&=2 \sum_{j<k}^{n_{1}-1} \mathbb{E}\left(\mathbb{E}_{k} F_{n}-\mathbb{E}_{k+1} F_{n}\right)\left(\mathbb{E}_{j} \bar{F}_{n}-\mathbb{E}_{j+1} \bar{F}_{n}\right)+\sum_{k=0}^{n_{1}-1} \mathbb{E}\left|\mathbb{E}_{k} F_{n}-\mathbb{E}_{k+1} F_{n}\right|^{2}= \\
&=\sum_{k=0}^{n_{1}-1} \mathbb{E}\left|\mathbb{E}_{k} F_{n}-\mathbb{E}_{k+1} F_{n}\right|^{2} .
\end{aligned}
$$

Here we use the identity $\mathbb{E}\left(\mathbb{E}_{k} F_{n}-\mathbb{E}_{k+1} F_{n}\right)\left(\mathbb{E}_{j} \bar{F}_{n}-\mathbb{E}_{j+1} \bar{F}_{n}\right)=0$ for $j \neq k$.

Denote by $\mathbb{E}^{(k)}$ averaging over $\left\{a_{k j}\right\}_{j=k}^{n}$. Let $F_{n}^{(k)}=\left.F_{n}\right|_{\left\{a_{k j}=0\right\}_{j=1}^{n}}$. So we get

$$
\mathbb{E}\left|\mathbb{E}_{k} F_{n}-\mathbb{E}_{k+1} F_{n}\right|^{2}=\mathbb{E}\left|\mathbb{E}_{k}\left(F_{n}-\mathbb{E}^{(k+1)} F_{n}\right)\right|^{2} \leq \mathbb{E}\left|\mathbb{E}_{k}\left(F_{n}-F_{n}^{(k+1)}\right)\right|^{2}
$$

Taking into account the Schwarz inequality, we obtain $\mathbb{E}\left|\mathbb{E}_{k}\left(F_{n}-F_{n}^{(k)}\right)\right|^{2} \leq \mathbb{E} \mathbb{E}_{k}\left|\left(F_{n}-F_{n}^{(k+1)}\right)\right|^{2}=$ $\mathbb{E}\left|F_{n}-F_{n}^{(k+1)}\right|^{2}$. Due to the symmetry we have for all $k$

$$
\mathbb{E}\left|\mathbb{E}_{k} F_{n}-\mathbb{E}_{k+1} F_{n}\right|^{2} \leq \mathbb{E}\left|F_{n}-F_{n}^{(k+1)}\right|^{2}=\mathbb{E}\left|F_{n}-F_{n}^{(1)}\right|^{2}
$$


In order to estimate $\mathbb{E}\left|F_{n}-F_{n}^{(1)}\right|^{2}$, we introduce the matrix $A(t)$ :

$$
A_{i j}(t)=\left(\mathbf{1}_{i \in I_{\alpha, N}, j \notin I_{\alpha, N}}+\mathbf{1}_{i \notin I_{\alpha, N}, j \in I_{\alpha, N}}\right) \cdot\left(\mathbf{1}_{i \geq 1, j \geq 1} a_{i j}+t \cdot\left(1-\mathbf{1}_{i \geq 1, j \geq 1}\right) \cdot a_{i j}\right) .
$$

Also we introduce the functions

$$
R(t)=(z-i A(t))^{-1}, \quad F_{n}(t)=n_{1}^{-1} \sum_{j=1}^{n_{1}} \varphi\left(R_{j j}(t)\right) .
$$

Clearly, the following equality is true

$$
F_{n}-F_{n}^{(1)}=\int_{0}^{1} \frac{d}{d t} F_{n}(t) d t
$$

We can estimate $\frac{d}{d t} F(t)$ by the following way:

$$
\frac{d}{d t} F_{n}(t)=\frac{1}{n_{1}} \sum_{j=1}^{n_{1}} \sum_{k, l} \xi_{j}(t) R_{j k}(t) A_{k l}^{\prime}(t) R_{l j}(t)=\frac{2}{n_{1}} \sum_{j \in I_{\alpha, n}, k, l} \xi_{j}(t) R_{j 1}(t) a_{1 k}(t) R_{k j}(t)=2 H,
$$

where

$$
\begin{gathered}
\xi_{j}(t)=\frac{\partial}{\partial R_{j j}} \varphi\left(R_{j j}(t)\right) . \\
\mathbb{E}\left\{|H|^{2}\right\} \leq \frac{C_{3}^{2}}{4 n_{1}^{2}} \mathbb{E}\left\{\sum_{j, j^{\prime} \in I_{\alpha, n}, k, k^{\prime}} \alpha_{j} \alpha_{j}^{\prime}\left(\left|R_{j k}\right|^{2}+\left|R_{1 j}\right|^{2}\right)\left|a_{1 k}\right|\left(\left|R_{j^{\prime} k^{\prime}}\right|^{2}+\left|R_{1 j^{\prime}}\right|^{2}\right)\left|a_{1 k^{\prime}}\right|\right\} \leq \\
\leq \frac{C_{3}^{2}}{n_{1}^{2}} \mathbb{E}\left\{\alpha_{1}^{2}\right\} \mathbb{E}\left(\sum_{k}\left|a_{1 k}\right|^{2}\right) \leq \frac{C_{3}^{2}}{n_{1}^{2}|\Re z|^{4}} \mathbb{E}\left\{\alpha_{1}^{2}\right\} \cdot\left(n_{2} \mathbb{E}\left|a_{12}\right|+\left(n_{2} \mathbb{E} a_{12}^{2}\right)^{2}\right)
\end{gathered}
$$

Here we use the inequality

$$
\sum_{j}\left|R_{s j}\right|^{2}=\left[R R^{*}\right]_{s s} \leq \frac{1}{|\Re z|^{2}} .
$$

Using (4.3) and (4.4), we obtain

$$
\begin{aligned}
\mathbb{E}\left|F_{n}-F_{n}^{(1)}\right|^{2}=\mathbb{E}\left\{\left|\int_{0}^{1} \frac{d}{d t} F_{n}(t)\right|^{2}\right\} \leq & \int_{0}^{1} \mathbb{E}\left\{\left|\frac{d}{d t} F_{n}(t)\right|^{2}\right\} \leq \\
& \leq \frac{4 C_{3}^{2}}{n_{1}^{2}|\Re z|^{4}} \cdot \mathbb{E}\left\{\alpha_{1}^{2}\right\} \cdot\left(n_{2} \mathbb{E}\left|a_{12}\right|+\left(n_{2} \mathbb{E} a_{12}^{2}\right)^{2}\right) .
\end{aligned}
$$

In much the same way the following estimate can be proved

$$
\begin{aligned}
\mathbb{E}\left|F_{n}-F_{n}^{(n)}\right|^{2}=\mathbb{E}\left\{\left|\int_{0}^{1} \frac{d}{d t} F_{n}(t)\right|^{2}\right\} \leq & \int_{0}^{1} \mathbb{E}\left\{\left|\frac{d}{d t} F_{n}(t)\right|^{2}\right\} \leq \\
& \leq \frac{4 C_{3}^{2}}{n_{1}^{2}|\Re z|^{4}} \cdot \mathbb{E}\left\{\alpha_{1}^{2}\right\} \cdot\left(n_{2} \mathbb{E}\left|a_{12}\right|+\left(n_{2} \mathbb{E} a_{12}^{2}\right)^{2}\right) .
\end{aligned}
$$

Combining (4.1), 4.2), (4.5) we obtain (3.18). 
Proof of Proposition 11. Denote by $a^{(T)}$ truncation of $a$ with parameter $T$, i.e.

$$
a^{(T)}(\omega)=\left\{\begin{array}{ll}
a(\omega), & \text { if } a(\omega)<T, \\
T, & \text { otherwise }
\end{array} .\right.
$$

Here and below the notation with an upper index $(T)$ means that the function is defined for the matrix $A^{(T)}$ by the same way as it was done for $A$.

Similar to (3.27) and (3.28), we can obtain that for any $\varphi$ : $\|\varphi\| \leq 2$

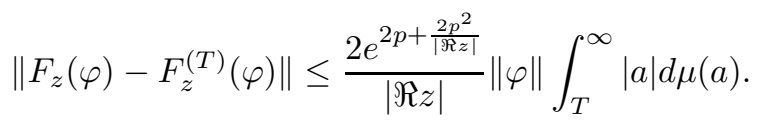

Combining (4.8) and (3.30), we obtain

$$
\forall z \in L(M) f^{(T)}(u, z) \underset{T \rightarrow \infty}{\stackrel{\|\|_{\mathcal{H}^{2}}}{\longrightarrow}} f(u, z) .
$$

Theorem 2 yields

$$
g_{\sigma_{p, \alpha}^{(T)}}(z)=\lim _{N \rightarrow \infty} \mathbb{E}\left\{g_{N, p, \alpha}^{(T)}(z)\right\},
$$

and for an arbitrary compact in $\mathbb{C}_{+}$the convergence is uniform.

Taking into account the resolvent identity and the Schwarz inequality, we obtain

$$
\left|\mathbb{E} g_{\sigma_{N, p, \alpha}^{(T)}}(z)-\mathbb{E} g_{\sigma_{N, p, \alpha}}(z)\right| \leq \frac{p}{N|\Im z|^{2}}\left(\int_{T}^{\infty} a^{2} d \mu(a)\right)^{1 / 2}
$$

Combining (4.9)-(4.11), we obtain

$$
g_{\sigma_{p, \alpha}}(z)=\lim _{N \rightarrow \infty} \mathbb{E}\left\{g_{N, p, \alpha}(z)\right\},
$$

and for an arbitrary compact in $\mathbb{C}_{+}$the convergence is uniform.

(2.12) is still valid because it doesn't require existence of $X_{4}$ (Just use Lemma1 for $\varphi(\zeta)=\zeta$, $\left.\alpha_{j}=1, C_{3}=1, n=N\right)$.

\section{References}

[1] M.Abramowitz, I.Stegun, Handbook of Mathematical Functions, Dover, N.Y., 1972

[2] M.Bauer and O.Golinelli. Random incidence matrices: spectral density at zero energy, Saclay preprint T00/087; cond-mat/0006472

[3] B. Bollobas Random Graphs Acad. Press (1985)

[4] M.Bauer and O.Golinelli. Random incidedence matrices: moments and spectral density, J.Stat. Phys. 103, 301-336, 2001

[5] Fan R.K. Chung, Spectral Graph Theory AMS (1997)

[6] D.M. Cvetković, M.Doob, and H.Sachs. Spectra of Graphs, Acad. Press (1980)

[7] S.N. Evangelou. Quantum percolation and the Anderson transition in dilute systems, Phys. Rev. B 27 (1983) 1397-1400

[8] S.N. Evangelou and E.N. Economou. Spectral density singularities, level statistics, and localization in sparse random matrices, Phys. Rev. Lett. 68 (1992) 361-364

[9] S.N. Evangelou. A numerical study of sparse random matrices, J. Stat. Phys. 69 (1992) 361-383 
[10] Y.V.Fyodorov, A.D.Mirlin. Strong eigenfunction correlations near the Anderson localization transition, Phys. Rev. B 55 (1997) R16001-R16004

[11] Ch. Godzil, G. Royle, Algebraic Graph Theory. Springer-Verlag, New York (2001)

[12] S. Janson, T. Łuczak, A. Rucinski, Random Graphs. John Wiley \& Sons, Inc. New York (2000)

[13] D. Jacobson, S.D. Miller, I. Rivin, and Z. Rudnick. Eigenvalue spacing for regular graphs, in: Emerging applications of number theory. Ed. D.A. Hejhal et al. Springer-Verlag (1999)

[14] Khorunzhy O., Shcherbina M., and Vengerovsky V. Eigenvalue distribution of large weighted random graphs, J. Math. Phys. 45 N.4: (2004), 1648-1672.

[15] A. Khorunzhy, B. Khoruzhenko, L. Pastur and M. Shcherbina. The Large-n Limit in Statistical Mechanics and Spectral Theory of Disordered Systems. Phase transition and critical phenomena.v.15, p.73, Academic Press, 1992

[16] M.L.Mehta: Random Matrices. New York: Academic Press, 1991

[17] A.D.Mirlin, Y.V.Fyodorov. Universality of the level correlation function of sparce random matrices, J.Phys.A:Math.Jen.24, (1991), 2273-2286.

[18] G.J. Rodgers and A.J. Bray. Density of states of a sparse random matrix, Phys.Rev.B 37, (1988), 3557-3562.

[19] G.J. Rodgers and C. De Dominicis. Density of states of sparse random matrices, J.Phys.A:Math.Jen.23, (1990), 1567-1566.

[20] V. Vengerovsky. Eigenvalue Distribution of a Large Weighted Bipartite Random Graph, JMPAG 10-2 (2014), 240-255.

[21] E.P.Wigner. On the distribution of the roots of certain symmetric matrices, Ann.Math. 67: (1958), 325-327. 